\section{Obstetric complications and schizophrenia ${ }^{\dagger}$}

\author{
Two case-control studies based on structured obstetric \\ records
}

R. E. KENDELL, K. McINNENY, E. JUSZCZAK and M. BAIN
Many case-control and a few cohort studies have suggested that a history of obstetric complications is more common in people with schizophrenic illnesses than in siblings or matched controls. Geddes \& Lawrie (1995) performed a meta-analysis of 20 case-control and two cohort studies and calculated that subjects who subsequently developed schizophrenia were twice as likely to have been exposed to obstetric complications of varied kinds as controls. Shortly afterwards we conducted a case-control study which also showed a highly significant $(P<0.001)$ excess of complications of both pregnancy and delivery in the probands with schizophrenia (Kendell et al, 1996). It is widely assumed that these obstetric complications contribute to the aetiology of schizophrenia and that anoxic damage to the foetal brain, either in late pregnancy or at the time of delivery, is the pathogenic mechanism. The evidence for these assumptions is not strong, however, mainly because most studies had to rely on a global index of obstetric complications because they had too few subjects to study individual complications.

The two case-control studies reported here were designed as an extension of our 1996 study. That had been based on 115 schizophrenic-control pairs derived from all hospital deliveries in Scotland in 197174 and was then the largest published study based on obstetric data recorded at the time of birth. By re-examining the original 1971-74 birth cohort 4 years later, by which time many more would have developed schizophrenic illnesses, and conducting an analogous case-control comparison on the subsequent 1975-78 birth cohort, we hoped to obtain a sufficiently large number of matched schizophrenic-control pairs to be able to compare the incidence of individual obstetric complications in the two, and thereby determine whether the complications which were more common in the schizophrenia probands than the controls were indeed those likely to result in foetal anoxia.

\section{METHOD}

\section{Basic design}

The basic design of both studies was very similar to our original study (Kendell et al, 1996) and based on the obstetric data (Scottish Morbidity Record 2 or SMR2) and psychiatric data (Scottish Morbidity Record 4 or SMR4) collected by the Information and Statistics Division (ISD) of the National Health Service in Scotland over the past 30 years. First, all individuals born between 1 January 1971 and 31 December 1978 admitted to a psychiatric hospital or unit anywhere in Scotland with a discharge diagnosis of schizophrenia for their most recent admission were identified from the psychiatric (SMR4) files. Schizophrenia was defined until 31 March 1996 by the World Health Organization's (WHO's) ICD-9 codes 295.0-295.9 (WHO, 1978). Thereafter it was defined by ICD-10 codes F20.0-20.3 and F20.5-20.9, i.e. omitting post-schizophrenic depression, F20.4 (WHO, 1992). At the time the files were examined in October 1997 almost all 1996 discharge data and some 1997 data had been received by the ISD from the psychiatric hospitals. A linkage was established between these SMR4 records and the national SMR2 file for the period 1971-78 and the Registrar General's birth records for the period 1974-78 (earlier birth records were not available in electronic form). These birth records were helpful because they contained demographic information about both the baby and the mother which made it easier to decide whether an SMR2 record, which does not give the baby's forenames, matched an SMR4 record. The linkage involved probability matching (Kendrick \& Clarke, 1993) which pulls together records belonging to the same individual with $98-99 \%$ accuracy. All doubtful links for births in 1971-73 were checked by hand using the microfiches of the original birth certificate in order to decide whether an SMR2 record related to the same person as the SMR4 record.

\section{Obstetric data}

SMR2 data consist of fairly extensive demographic and medical information about the mother and salient features of the current pregnancy, birth and puerperium. The clinical information was

†See pp. 523-530, this issue. 
originally recorded on a structured antenatal record sheet (by the relevant obstetrician) and on a similarly structured delivery record (by the midwife or obstetrician responsible for the delivery). This information was then transcribed onto the ISD's SMR2 form (by a medical records clerk or the obstetrician) when the mother left hospital and subsequently transferred to magnetic tape. In addition to the mandatory ratings there was provision on the form for recording other less common complications of pregnancy, delivery or the puerperium using four digit ICD-8 code numbers. Because the content and layout of the SMR2 form was changed on 1 January 1975 , and also because our original study had been based on individuals born in 1971-74, 1971-74 and 1975-78 births were analysed separately. As before, the SMR2 records of the probands with schizophrenia were individually matched with controls who had never been admitted to a Scottish psychiatric unit with a discharge diagnosis of either schizophrenia or an affective psychosis on six different variables - the obstetric unit of birth, gender, date of birth $( \pm 2$ months), maternal age ( \pm 2 years), maternal parity (first baby $v$. second or subsequent baby) and father's occupation (manual $v$. non-manual). Twins and singletons were also matched separately. A composite variable birth weight less than $2500 \mathrm{~g}$ and gestational age less than 37 weeks was added to the SMR2 variables originally chosen for analysis because of the recent finding by Jones et al (1998) that this combination, indicating normal intra-uterine growth but premature delivery, was a risk factor for schizophrenia. Small for gestational age (below the 5th percentile) was also added.

SMR2 records were found for 505 $(56 \%)$ of the 902 people with schizophrenia born in 1971-78 originally identified from the SMR4 file. The main reasons for failure to identify an SMR2 record were birth outside Scotland or home delivery (in $197124 \%$ of births were either home births or in small obstetric units not supplying SMR2 returns, but this had fallen to $2 \%$ by 1978 ). Changes of surname on adoption, coding errors and failure of the psychiatric hospital to record the original maiden name of married women are other possibilities, but the fact that the male: female ratio was not significantly higher in the 505 probands than in the 397 potential probands for whom no SMR2 record could be found suggests that change of surname on marriage was not an important cause of failure.

\section{Demographic data}

Vital demographic information (mainly the father's occupation) was missing from 34 obstetric records, which reduced the total available for matching from 505 to 471 , but because so many potential controls were available (there were nearly 500000 hospital deliveries in Scotland in 1971-78) it was possible to match all but 19 of these on all the six variables described above. We therefore ended up with 452 matched pairs - 296 born in 1971-74 and 156 born in 1975-78.

At the time the SMR4 data were originally extracted from file (October 1997) the age of the 1971-74 probands ranged from 22 to 26 years and $78.7 \%$ were male. The age of the 1975-78 probands ranged from 18 to 22 years and $75.6 \%$ were male. The high percentage of males is a consequence of the earlier age of onset and hospital admission in male than female patients with schizophrenia.

\section{Statistical analysis}

A standard case-control analysis of matched case-control sets was carried out using SPSS for Windows and STATA (Breslow \& Day, 1980; Stata, 1997). Odds ratios, 95\% confidence intervals (CIs) and $P$ values were calculated using conditional logistic regression. Probands and controls were also compared using the paired $t$-test for the normally distributed continuous variables birthweight, antenatal haemoglobin and gestational age and the Wilcoxon signed ranks test for the non-normally distributed variable duration of labour.

\section{RESULTS}

\section{7|-74 births}

The results of the proband-control comparisons are shown in Table 1 . None of the differences is large and not a single complication of either pregnancy or delivery is significantly more common in the probands than in their controls. Two complications of pregnancy - maternal abdominal or pelvic X-ray and admission to hospital - are more common in controls $(P<0.05)$ and maternal antenatal haemoglobin is also lower in controls $(P=0.05)$. Even though there were no differences between the probands and their controls in the total numbers of complications of pregnancy and delivery recorded, all the complications recorded by their ICD-8 code numbers were compared individually. There was a significant difference for one item only - delivery complicated by malpresentation of the foetus, which was significantly more common in the controls (11 v. 3; $P<0.05$ ). Proband-control comparisons were also carried out for males and females separately. No significant differences emerged apart from abdominal or pelvic X-ray in pregnancy and antenatal haemoglobin. For both variables the difference described above was restricted to females.

\section{5-78 births}

The redesigned SMR2 form introduced on 1 January 1975 provided less information about the mother's health during pregnancy than the original 1971 form, but more about the delivery and the newborn child. In particular, it distinguished between elective and emergency Caesarean sections and recorded the Apgar score 5 minutes after birth. The results of the proband-control comparisons are shown in Table 2. Again, the two are very similar, though this time there are two variables which are significantly more common in those with schizophrenia-emergency Caesarean section $(P=0.05)$ and a duration of labour of over 12 hours $(P=0.04)$. The mean duration of labour is also longer in the probands than in the controls $(P=0.05)$.

As before, all of the complications recorded by their ICD- 8 code numbers were compared individually as well as corporately. There were significant differences for only two. Delivery complicated by malpresentation of the foetus was more common in the probands $(6 v .0 ; P<0.05)$ and laceration of the perineum was more common in the controls $(9 v .2 ; P<0.05)$. Again, separate proband-control comparisons were carried out for males and females. None of the differences described above was statistically significant in either of these single gender comparisons, nor did any new significant proband-control differences emerge.

\section{Reappraisal}

The results described above are completely different from those obtained in our previous study of the 1971-74 birth cohort (Kendell et al, 1996). Indeed, they are so different they are incompatible. The 1996 
Table I 197I-74 births. Complications of pregnancy, delivery and the puerperium. Comparison of schizophrenia probands and controls (296 matched pairs)

\begin{tabular}{|c|c|c|c|c|c|}
\hline & Probands & Controls & Odds ratio & $95 \% \mathrm{Cl}$ & $P$ value \\
\hline \multicolumn{6}{|l|}{ Dichotomous variables } \\
\hline Pre-existing maternal illness & 9 & 9 & 1.00 & $0.40-2.52$ & 1.00 \\
\hline \multicolumn{6}{|l|}{ Complications of pregnancy } \\
\hline Anaemia & 6 & II & 0.44 & $0.14-1.44$ & 0.18 \\
\hline Urinary infection & $\mathrm{II}$ & 7 & 1.67 & $0.61-4.59$ & 0.32 \\
\hline Haemorrhage & II & 4 & 2.75 & $0.88-8.64$ & 0.08 \\
\hline Rhesus antibodies & 6 & 1 & 6.00 & $0.72-49.84$ & 0.10 \\
\hline Abdominal or pelvic $X$-ray & 21 & 36 & 0.53 & $0.30-0.96$ & 0.04 \\
\hline Blood transfusion & 9 & 9 & 1.00 & $0.35-2.85$ & 1.00 \\
\hline Pre-eclampsia & 30 & 27 & 1.12 & $0.65-1.92$ & 0.68 \\
\hline Admission to hospital & 46 & 65 & 0.63 & $0.4 I-0.98$ & 0.04 \\
\hline Any complication' & 71 & 64 & 1.18 & $0.77-1.82$ & 0.44 \\
\hline \multicolumn{6}{|l|}{ Complications of delivery } \\
\hline Abnormal presentation & 6 & 14 & 0.38 & $0.14-1.08$ & 0.07 \\
\hline Artificial rupture of membranes & $|2|$ & III & 1.18 & $0.83-1.69$ & 0.36 \\
\hline Oxytocics & 67 & 70 & 0.89 & $0.58-1.36$ & 0.59 \\
\hline Forceps delivery & 25 & 34 & 0.63 & $0.33-1.19$ & 0.15 \\
\hline Caesarean section & 18 & 20 & 0.88 & $0.43-1.79$ & 0.72 \\
\hline Non-spontaneous delivery & 44 & 58 & 0.63 & $0.38-1.05$ & 0.08 \\
\hline Baby small for gestational age & 22 & 25 & 0.88 & $0.49-1.57$ & 0.66 \\
\hline Birthweight $<2500 \mathrm{~g}$, gestation $<37$ weeks & 8 & 5 & 1.60 & $0.52-4.89$ & 0.41 \\
\hline Baby detained in hospital & 28 & 38 & 0.68 & $0.39-1.18$ & 0.17 \\
\hline Any complication' & 67 & 61 & 1.17 & $0.75-1.84$ & 0.49 \\
\hline \multicolumn{6}{|l|}{ Puerperium } \\
\hline Any complication' & 21 & 22 & 0.94 & $0.48-1.86$ & 0.86 \\
\hline \multicolumn{6}{|l|}{ Continuous variables (mean (s.d.)) } \\
\hline Antenatal haemoglobin $(\mathrm{g})$ & $11.40(1.14)$ & $11.21(1.15)$ & & $t=1.94$ & 0.05 \\
\hline Duration of pregnancy (weeks) & $39.55(2.19)$ & $39.48(2.00)$ & & $t=0.36$ & 0.71 \\
\hline Duration of labour (hours) & $7.79(5.87)$ & $7.40(6.30)$ & & $z=1.36$ & 0.17 \\
\hline Birth weight $(g)$ & $3295(522)$ & $3339(484)$ & & $t=1.08$ & 0.30 \\
\hline
\end{tabular}

I. These items represent the total number of (mostly rare) complications recorded on the Scottish Morbidity Record 2 form by their ICD- 8 code numbers. For this reason they cannot be related to any combination of the other variables listed in the table.

study, which was based on the 115 probands who had developed a schizophrenic illness by the end of 1992, had produced a strikingly higher incidence of complications both of pregnancy and delivery in the probands than in the controls $(P<0.001$ in each case). Four years later, by which time more than twice as many members of the birth cohort had developed schizophrenic illnesses, those probandcontrol differences had entirely disappeared. Although there is evidence that a raised incidence of obstetric complications is more common in people developing a schizophrenic illness before the age of 22 than in those with a later age of onset (Verdoux et al, 1997) this could hardly account for a change of such magnitude. Moreover, there were few significant proband-control differences in the 1975-78 birth cohort, who were the same age in 1996 as the original 1971-74 cohort had been in 1992.

A comparison of the incidence of obstetric complications in both the probands and the controls of the 1971-74 birth cohort in the original 1996 study and the present one showed that the incidence of complications in the probands with schizophrenia was similar in both. The discrepancy was in the controls, who had a much lower incidence of complications, both of pregnancy and delivery, in the 1996 comparison than in the present one. We therefore compared the frequency of selected complications in four populations: the controls from our original 1996 study of the 1971-74 birth cohort; the controls from our present studies of both the 1971-74 and 1975-78 birth cohorts; and in all 500000 hospital deliveries in 1971-78 after excluding multiple births, neonatal deaths and the probands with schizophrenia and affective disorders in our present studies. The results are shown in Table 3, and it is apparent that the original 1971-74 controls are anomalous with substantially fewer complications of pregnancy, delivery and the puerperium than the other three populations.

Initially, this seemed inexplicable because in all three studies (the original study of the 1971-74 birth cohort and the present studies of the 1971-74 and 1975-78 birth cohorts) not only had probands and controls been closely matched on the same six variables, but an almost identical computer program had been used to generate the controls. In a bid to identify the cause of the discrepancy an attempt was made to repeat the original (1996) study of the 1971-74 birth cohort. 
Table 2 1975-78 births. Complications of pregnancy, delivery and the puerperium. Comparison of schizophrenia probands and controls (I56 matched pairs)

\begin{tabular}{|c|c|c|c|c|c|}
\hline & Probands & Controls & Odds ratio & $95 \% \mathrm{Cl}$ & $P$ value \\
\hline \multicolumn{6}{|l|}{ Dichotomous variables } \\
\hline Pre-existing maternal illness & 5 & 3 & 1.67 & $0.40-6.97$ & 0.48 \\
\hline \multicolumn{6}{|l|}{ Complications of pregnancy } \\
\hline Anaemia & 5 & 2 & 2.50 & $0.49-12.89$ & 0.27 \\
\hline Urinary infection & 6 & 12 & 0.50 & $0.19-1.33$ & 0.17 \\
\hline Haemorrhage & 5 & 8 & 0.63 & $0.20-1.91$ & 0.41 \\
\hline Pre-eclampsia & 19 & 22 & 0.85 & $0.45-1.62$ & 0.62 \\
\hline Admission to hospital & $4 I$ & 34 & 1.28 & $0.76-2.16$ & 0.36 \\
\hline Any complication' & 47 & 46 & 1.04 & $0.61-1.78$ & 0.89 \\
\hline \multicolumn{6}{|l|}{ Complications of delivery } \\
\hline Abnormal presentation & II & 9 & 1.25 & $0.49-3.17$ & 0.64 \\
\hline Artificial rupture of membranes & 60 & 53 & 1.27 & $0.76-2.12$ & 0.36 \\
\hline Oxytocics & 46 & 42 & 1.17 & $0.67-2.05$ & 0.57 \\
\hline Breech delivery & 3 & 2 & 1.50 & $0.25-8.98$ & 0.66 \\
\hline Forceps delivery & 17 & 21 & 0.78 & $0.39-1.56$ & 0.48 \\
\hline Caesarean section - emergency or elective & 16 & 11 & 1.50 & $0.67-3.34$ & 0.32 \\
\hline Emergency Caesarean section & 11 & 3 & 3.67 & $1.02-13.14$ & 0.05 \\
\hline Non-spontaneous delivery & 40 & 36 & 1.17 & $0.67-2.05$ & 0.57 \\
\hline Apgar score 7 or 8 & 16 & 25 & 0.45 & $0.20-1.03$ & 0.06 \\
\hline Apgar score under 7 & 6 & 6 & 0.73 & $0.20-2.69$ & 0.64 \\
\hline Baby small for gestational age & 12 & 6 & 2.20 & $0.76-6.33$ & 0.14 \\
\hline Birthweight $<2500 \mathrm{~g}$, gestation $<37$ weeks & 4 & 5 & 0.80 & $0.21-2.98$ & 0.74 \\
\hline Admission to special care baby unit & 36 & 39 & 0.90 & $0.53-1.52$ & 0.69 \\
\hline Any complication' & 48 & 40 & 1.38 & $0.79-2.42$ & 0.26 \\
\hline \multicolumn{6}{|l|}{ Puerperium } \\
\hline Any complication' & II & 6 & 2.67 & $0.71-10.05$ & 0.15 \\
\hline \multicolumn{6}{|l|}{ Continuous variables (mean (s.d.)) } \\
\hline Duration of pregnancy (weeks) & $39.44(1.8 I)$ & $39.63(1.54)$ & & $t=1.00$ & 0.32 \\
\hline Duration of labour (hours) & $7.40(5.36)$ & $6.30(3.97)$ & & $z=2.11$ & 0.04 \\
\hline Birth weight (g) & $3264(480)$ & $3303(482)$ & & $t=0.72$ & 0.47 \\
\hline
\end{tabular}

I. These items represent the total number of (mostly rare) complications recorded on the Scottish Morbidity Record 2 form by their ICD- 8 code numbers. For this reason they cannot be related to any combination of the other variables listed in the table.

All the original 115 cases were re-identified and each stage of the original analysis repeated. It eventually became clear that, because the original matching program took the first subject in the SMR2 master file who matched each successive proband on the requisite six variables as that proband's control rather than the closest matching subject, the order in which individual births appeared in the SMR2 file was critical. If the order was left as it stood in the SMR2 master file, which reflected the order in which returns had been made by individual obstetric units to the ISD in
Edinburgh, the incidence of complications in the controls was low (Column 4 in Table 4) and almost identical to the results reported in 1996 (Column 2 in Table 4).

If, on the other hand, the SMR2 file was re-sorted so that, for each obstetric unit, all subjects appeared in sequence according to

Table 3 Frequency of selected obstetric complications in four normal or control populations

\begin{tabular}{lcccc}
\hline & $\begin{array}{c}\text { Original I97I-74 controls } \\
(n=115)(\%)\end{array}$ & $\begin{array}{c}\text { Present 197I-74 controls } \\
(n=296)(\%)\end{array}$ & $\begin{array}{c}\text { Present 1975-78 controls } \\
(n=156)(\%)\end{array}$ & $\begin{array}{c}\text { All I97I-78 SMR2 } \\
\text { records }(n=495 \text { 536) }\end{array}$ \\
\hline Antepartum haemorrhage & 0 & 1.4 & 5.1 & 3.6 \\
Pre-eclampsia & 1.7 & 9.1 & 14.1 & 12.0 \\
Complications of pregnancy & 7.0 & 21.6 & 29.5 & 27.0 \\
Complications of delivery & 0.9 & 20.6 & 25.6 & 24.7 \\
Complications of the puerperium & 2.6 & 7.4 & 3.8 & 6.6 \\
\hline
\end{tabular}

SMR2, Scottish Morbidity Record 2. 
Table 4 Reconstruction and re-analyses of the original (1996) study of the 1971-74 birth cohort

\begin{tabular}{|c|c|c|c|c|c|c|}
\hline \multirow[t]{3}{*}{ Matched pairs } & \multicolumn{2}{|c|}{$\begin{array}{l}\text { Original study as } \\
\text { published }\end{array}$} & \multicolumn{2}{|c|}{$\begin{array}{l}\text { Repeat analysis, control } \\
\text { file unsorted }\end{array}$} & \multicolumn{2}{|c|}{$\begin{array}{c}\text { Repeat analysis, control file sorted } \\
\text { by date of birth }\end{array}$} \\
\hline & \multicolumn{2}{|c|}{$(n=115)$} & \multicolumn{2}{|c|}{$(n=I 10)$} & \multicolumn{2}{|c|}{$(n=107)$} \\
\hline & Probands & Controls & Probands & Controls & Probands & Controls \\
\hline Pre-existing maternal illness & 5 & 2 & 4 & 2 & 4 & 2 \\
\hline \multicolumn{7}{|l|}{ Complications of pregnancy } \\
\hline Anaemia & 7 & I & 7 & I & $5^{*}$ & 0 \\
\hline Urinary infection & 4 & 0 & 4 & 0 & 4 & 2 \\
\hline Haemorrhage & 3 & 0 & 3 & 0 & 3 & 2 \\
\hline Rhesus antibodies & 7 & 4 & 7 & 3 & 3 & 5 \\
\hline Abdominal or pelvic $X$-ray & 14 & 12 & 13 & II & II & 13 \\
\hline Blood transfusion & 4 & 2 & 4 & 2 & 4 & 2 \\
\hline Pre-eclampsia & $10 *$ & 2 & 9* & 2 & 8 & 14 \\
\hline Admission to hospital & 26 & 22 & 24 & 20 & 22 & 19 \\
\hline Any complication & $34 * * *$ & 8 & $32 * * *$ & 7 & 29 & 20 \\
\hline \multicolumn{7}{|l|}{ Complications of delivery } \\
\hline Abnormal presentation & 4 & I & 3 & 0 & 3 & 2 \\
\hline Artificial rupture of membranes & 42 & 50 & 40 & 46 & 39 & 47 \\
\hline Oxytocics & 22 & 30 & 20 & 27 & 20 & 23 \\
\hline Forceps delivery & 9 & 2 & 8 & 2 & 7 & 12 \\
\hline Caesarean section & 4 & 2 & 4 & 2 & 4 & 5 \\
\hline Non-spontaneous delivery & $14 *$ & 4 & $13^{*}$ & 4 & 12 & 17 \\
\hline Baby detained in hospital & $18 *$ & 6 & $18 * *$ & 6 & 17 & 13 \\
\hline Any complication & $18 * * *$ & I & $18^{* * *}$ & 0 & $18 *$ & 31 \\
\hline \multicolumn{7}{|l|}{ Puerperium } \\
\hline Any complication & 7 & 3 & 6 & 2 & 6 & 2 \\
\hline
\end{tabular}

* Proband-control difference significant at $5 \%$ level, $P=0.05$.

** Proband-control difference significant at $1 \%$ level, $P=0.01$.

*** Proband-control difference significant at $0.1 \%$ level, $P=0.001$.

the date of delivery (Column 6 in Table 4) before matching on the six variables was carried out, the incidence of complications in the controls was far higher and all the significant proband-control differences disappeared. Indeed, in the case of complications of delivery they were reversed.

We presume that the explanation of this striking and unexpected finding is that the SMR2 forms of straightforward, uncomplicated deliveries are generally completed, returned to the ISD and logged in the master file soon after the delivery, whereas the SMR2 forms of complicated deliveries tend to be returned to the ISD later on, either because the mother and/or baby remain in hospital longer after delivery or for other reasons. As a result, for any given date of birth, uncomplicated deliveries tend to precede complicated deliveries in the master file and, unless the master file is re-sorted first to remove this bias, uncomplicated deliveries tend to be selectively picked as controls. We also have some evidence to support this assumption. We compared the lengths of stay in the obstetric unit of the selected controls in the repeat of the 1996 study (i.e. using the SMR2 master file as it stood-Column 4 in Table 4) with the average length of stay of all other potential controls (i.e. women on the SMR2 master file who also matched the proband on the six variables). Six of the selected controls had the same length of stay as the average of the other potential controls, 26 had a longer stay and 77 (three times as many) had a shorter stay in the obstetric unit. We therefore conclude that the casecontrol comparisons shown in Tables 1 and 2 are valid and that the results of our original study of the 1971-74 cohort (Kendell et al, 1996) are seriously misleading and should be ignored.

\section{DISCUSSION}

\section{Summary of results}

If it is accepted that, for the reason described above, the published results of our original 1996 study were seriously misleading and that the results of the case-control studies of the 1971-74 and 1975-78 birth cohorts described here are accurate and dependable - and we are convinced that this is so - we have now failed to find any substantial evidence that the incidence of obstetric complications in people who subsequently develop schizophrenia is raised. The 1971-74 study, which was based on nearly 300 case-control pairs, did not identify a single complication, either of pregnancy or delivery, which was significantly more common in the probands than the controls. The 1975-78 study, which was based on only half the number of matched pairs because the cohort was 4 
years behind in its passage through the risk period for schizophrenia, produced three variables which were significantly more common in the probands - emergency Caesarean section, a labour lasting over 12 hours (and also a longer mean duration of labour) and delivery complicated by malpresentation of the foetus. Malpresentation is almost certainly a chance finding because it was significantly more common in the controls in the 1971-74 comparison. The other two may be genuine differences, particularly as they have been found before by other investigators. They are also both likely to be associated with foetal anoxia. On the other hand, they may be chance findings. Both were only significant at the $5 \%$ level, neither emerged in either males or females when these were analysed separately, and as those with schizophrenia and the controls were compared on some 40 variables it is likely that some significant differences would emerge at the $5 \%$ level by chance alone. (The SMR2 form did not distinguish elective and emergency Caesarean sections until 1975, but in the 197174 comparison total Caesarean sections were slightly more common in the controls than in the probands $(20 v$. 18). There was, though, a modest excess of probands with a labour lasting over 12 hours in the 1971-74 comparison (55 v. 46, $P>0.30)$.)

\section{Comparison with previous studies}

This failure to find substantial differences between patients with schizophrenia and controls is at variance with most of the existing literature and, in particular, with the meta-analysis of Geddes \& Lawrie (1995). However, Geddes \& Lawrie were careful to point out that there was a gap in their funnel plot in the region of small negative studies, and that the association they found between obstetric complications and schizophrenia might have been inflated by both selection and publication biases. It is also important to note that neither of the two cohort studies in their meta-analysis produced a significant schizophrenia-control difference; and that these and their 16 case-control studies only contained a total of 1000 subjects with schizophrenia, whereas the two reported here contained nearly half that number. Moreover, most of those 1000 subjects with schizophrenia were derived from studies with serious methodological deficiencies. In several obstetric information had been obtained retrospectively, from mothers or other family members or even from adult psychiatric case notes, and in others the matching of patients and controls was imperfect.

\section{Other recent studies}

Recently, three substantial and welldesigned studies based on obstetric records have been published. Jones et al (1998) reported a 28-year follow-up of a large Finnish birth cohort whose intra-uterine development and births had been systematically documented in 1966. Seventy-six cases of well-validated DSM-III-R schizophrenia were identified in this cohort, making it possible to identify obstetric risk factors for the development of schizophrenia. Low birth weight and the combination of low birth weight and short gestation were both significantly associated with subsequent schizophrenia. So too were placental abruption and an Apgar score of less than 8 at 1 minute. But no association was found with any of the common complications of pregnancy and delivery and the authors concluded that most of the relationships they found 'appeared to be due largely to characteristics of the child, not the delivery'.

The other two studies (Hultman et al, 1999; Dalman et al, 1999) were very similar both to our study and to one another. Both were based on a Swedish national cohort of births in the 1970s, used obstetric information recorded in a standard form at the time the infant left hospital, and identified members of the cohort who subsequently developed schizoprenic illnesses from a national register of admissions to psychiatric hospitals. Hultman et al identified 167 probands with schizophrenia (aged 15-21 years at the time of their first psychiatric admission) in the 1973-79 birth cohort and compared each of them with five controls. They studied a total of 44 variables and found schizophrenia to be significantly associated with multi-parity (odds ratio 2.0) and maternal bleeding during pregnancy (odds ratio 3.5 ). They also found significant associations in males, but not in females, for birth weight low for gestational age, coming fourth or later in a sibship and maternal bleeding in pregnancy. Dalman et al identified 238 probands with schizophrenia from a more restricted birth cohort (1973-77), presumably because they searched the psychiatric register at a later date when the cohort was somewhat older (up to age 22). They also compared their probands, not with individually matched controls, but with the rest of the birth cohort (over 500000 individuals) using logistic regression analysis. Despite the fact that Dalman et al (1999) and Hultman et al (1999) were using the same Swedish national data, and that most of their probands with schizophrenia must have been the same people, the results reported by Dalman and her colleagues are surprisingly different. They studied 32 obstetric variables and, after adjusting for psychotic illness in the mothers, found several of these to be associated with an increased risk of schizophrenia. Preeclampsia (relative risk 2.5) was the most important risk factor, followed by vacuum extraction (relative risk 1.7) and congenital malformations (relative risk 2.4). Their final conclusion was that foetal malnutrition in utero, hypoxia at the time of delivery and prematurity all contribute independently to the aetiology of schizophrenia, and that malnutrition is relatively more important in males and prematurity more important in females.

\section{Strengths and weaknesses of this study}

This present investigation has the same strengths and weaknesses as most other studies based on routinely recorded clinical data. The obstetric (SMR2) data were fairly comprehensive and recorded in a set numerical format within a few days of delivery. They therefore could not have been influenced by subsequent events or our expectations. Their existence also enabled us to obtain larger numbers of matched pairs than any previous case-control comparison. On the other hand, information about their accuracy and reliability is limited. Some information about the latter is available from a study of 1991 SMR2 data by the staff of the ISD in which a random sample of 651 SMR2 returns were compared with the patients' hospital records. Incomplete demographic information was a common problem (the woman's maiden name, for example, was not recorded in $9 \%$ of returns) but inaccurate recording of obstetric information was uncommon (mode of delivery, birthweight and Apgar score were incorrectly recorded in only $0.9 \%, 0.5 \%$ and $0.6 \%$ of records, though duration of labour was incorrect in $3.7 \%$ ). No similar study has yet been carried out on the psychiatric (SMR4) data. Discharge diagnoses are more likely to be reliable and valid than admission diagnoses, but 
these were purely clinical diagnoses and not based on operational criteria. Low reliability will, of course, tend to diminish proband-control differences. It could hardly, though, obscure those differences completely unless they were in reality very modest. The availability of the obstetric records of over 500000 hospital births for the 8-year period 1971-78 meant that the probands could be closely matched with controls on all relevant variables without any significant loss (only 19 of 471 probands remained unmatched, a $4 \%$ loss). The fact that births were ranked by date of birth before the controls were identified, and that the program employed picked the closest match for each proband rather than the first, also ensured that the matching process was not subject to the serious bias which, with hindsight, distorted the results of our original 1996 study.

\section{Implications}

Despite their methodological strengths and substantial numbers of probands it is difficult to reconcile the four most recent investigations of the role of obstetric complications in the genesis of schizophrenia (Jones et al, 1998; Hultman et al, 1999; Dalman et al, 1999 and this present study) with one another. Taken as a whole they suggest that the incidence of obstetric complications is raised in people with schizophrenia, but that the magnitude of this increase is much less than the 2:1 excess indicated by the earlier literature summarised by Geddes \& Lawrie (1995). Nor is there any agreement on which individual obstetric variables are the most important risk factors, and therefore no clear leads to the pathogenic mechanisms potentially involved. It should also be noted that our study, which found fewer differences between probands and controls than any previous comparison, had a larger number of probands with schizophrenia than any of its predecessors.

At this stage, therefore, there are only two safe conclusions. First, the relationship between obstetric complications and schizophrenia is less firmly established than has recently been assumed. Second, a further meta-analysis is needed, restricted to investigations based on a clearly defined

\section{CLINICAL IMPLICATIONS}

- The increased incidence of obstetric complications in schizophrenia is less firmly established than has recently been assumed.

- A further meta-analysis of studies of this relationship is needed, restricted to methodologically sound investigations.

Apparently minor procedural differences may result in successive analyses of the same data producing radically different results.

\section{LIMITATIONS}

Little information is available about the reliability of the routinely recorded obstetric data on which the study was based.

Subjects with schizophrenia were identified by clinical diagnosis at the time of discharge from inpatient care.

- All subjects were under the age of 27 years. The cohort had therefore not passed through the full risk period for schizophrenia.

R. E. KENDELL, FRSE, Edinburgh University Department of Psychiatry, Royal Edinburgh Hospital, Edinburgh; K. McINNENY, BA, E. JUSZCZAK, MSc, M. BAIN, MFPHM, Information and Statistics Division, National Health Service in Scotland, Edinburgh

Correspondence: Dr R. E. Kendell, 3 West Castle Road, Edinburgh EHI0 5AT

(First received 12 January 1999, final revision 25 October 1999, accepted 26 October 1999)

birth cohort and obstetric data recorded at the time of delivery.

\section{REFERENCES}

American Psychiatric Association (1987) Diagnostic and Statistical Manual of Mental Disorders (3rd edn, revised) (DSM-III-R). Washington, DC: APA.

Breslow, N. E. \& Day, N. E. (1980) The analysis of case-control studies. Statistical Methods in Cancer Research, Vol. I. IARC Scientific Publications, No. 32 Lyon: International Agency for Research on Cancer

Dalman, C., Allebeck, P., Cullberg, J., et al (1999) Obstetric complications and the risk of schizophrenia: a longitudinal study of a national birth cohort. Archives of General Psychiatry, 56, 234-240.

Geddes, J. R. \& Lawrie, S. M. (1995) Obstetric complications and schizophrenia: a meta-analysis. British Journal of Psychiatry, 167, 786-793.

Hultman, C. M., Sparén, P., Takei, N., et al (1999) Prenatal and perinatal risk factors for schizophrenia, affective psychosis, and reactive psychosis of early onset: case-control study. British Medical Journal, 318 $421-426$
Jones, P. B., Rantakallio, P., Hartikainen, A., et al (1998) Schizophrenia as a long-term outcome of pregnancy, delivery and perinatal complications: a 28 year follow-up of the 1966 North Finland general population birth cohort. American Journal of Psychiatry I55, 355-364

Kendell, R. E., Juszczak, E. \& Cole, S. K. (1996) Obstetric complications and schizophrenia: a case control study based on standardised obstetric records. British Journal of Psychiatry, 168, 556-561.

Kendrick, S. \& Clarke, J. (1993) The Scottish record linkage system. Health Bulletin, 5I, 72-79.

Stata (1997) Stata Statistical Software: release 5.0. College Station, TX

Verdoux, H., Geddes, J. R., Takei, N., et al (1997) Obstetric complications and age at onset in schizophrenia: an international collaborative metaanalysis of individual patient data. American journal of Psychiatry, 154, 1220-1227.

World Health Organization (1978) Mental Disorders: Glossary and Guide to their Classification in Accordance with the Ninth Revision of the International Classification of Diseases. Geneva: WHO

- (1992) The ICD-10 Classification of Mental and Behavioural Disorders: Clinical Descriptions and Diagnostic Guidelines. Geneva: WHO 